\title{
Effect of frequency on multi-axial fretting fatigue damage behavior of Al-Zn-Mg alloys
}

\author{
Fu Xuemin ${ }^{1, a}$, Jiang Jiaxin ${ }^{2^{*}, b}$ and Yang Beilei ${ }^{3, c}$, Jiang Xiaosong ${ }^{4, d}$ \\ ${ }^{1}$ School of Mechanical Engineering, Chengdu Textile College, Chengdu 611731, P. R. China \\ ${ }^{2}$ School of Mechanical Engineering, Chengdu Textile College, Chengdu 611731, P. R. China \\ ${ }^{3}$ School of Materials Science and Engineering, Southwest Jiaotong University, Chengdu 610031, P. \\ R. China \\ ${ }^{4}$ School of Materials Science and Engineering, Southwest Jiaotong University, Chengdu 610031, P. \\ R. China \\ a573098007@qq.com, bjiangjiaxin2004@126.com, cybl11@qq.com, dxs jiang@126.com
}

Correspondence should be addressed to Jiang Jiaxin, jiangjiaxin2004@126.com

Keywords: frequency, Al-Zn-Mg alloy, fretting fatigue, damage behavior

\begin{abstract}
In this paper, effect of frequency on multi-axial fretting fatigue damage behavior of Al-Zn-Mg alloys is studied. Point contact fretting fatigue tests are used to research effect of frequency on multi-axial fretting fatigue lives, fretting wear area and fracture appearance. Based on experimental results of effect of frequency on multi-axial fretting fatigue of $\mathrm{Al}-\mathrm{Zn}-\mathrm{Mg}$ alloys, fretting fatigue damage behavior of $\mathrm{Al}-\mathrm{Zn}-\mathrm{Mg}$ alloys are intensively analyzed. Results showed that there exists serious stress concentration in fretting fatigue damage zone which due to fretting fatigue as the essence of frequency is chemical factors which is related to time. With frequency increases, fretting fatigue lives reduce nonlinearly and then increase in which lies in a break point in that existence of competitive relations between fatigue failure and surface abrasion.
\end{abstract}

\section{Introduction}

Fretting fatigue is a phenomenon which occurs between two contact surfaces with small-amplitude oscillatory motion, and it can also be described as a combination of two concomitant processes: fretting and fatigue [1-3]. This process leads to the micro-cracks of the surface, which reduce fatigue strength or make the failure of specimen. Fretting fatigue comprehensively exists in the junction of mechanic components and it has very serious consequences [1-3]. Al-Zn-Mg alloys are widely used as structural materials which are extensively applied to rail transit and other engineering projects [4-6]. Fretting fatigue damage is very common in these application areas and effect of frequency on multi-axial fretting fatigue damage behavior of Al-Zn-Mg alloys has not been studied deeply [7]. In this experimental study, multi-axial fretting fatigue behavior of $\mathrm{Al}-\mathrm{Zn}-\mathrm{Mg}$ alloys under different frequencies is presented to analyze fretting fatigue damage mechanism by researching characteristics of fretting fatigue life, fretting wear area and fracture appearance. Results showed that there exists serious stress concentration in fretting fatigue damage zone which due to fretting fatigue as the essence of frequency is chemical factors which is related to time. With frequency increases, fretting fatigue lives reduce nonlinearly and then increase in which lies in a break point in that existence of competitive relations between fatigue failure and surface abrasion.

\section{Experimental procedure}

This axial/torsional fretting fatigue test is conducted by MTS809 axial/torsional electro-hydraulic servo testing machine. Control frequency as the variable. The related test parameters is controlled and collected through data acquisition and control system connected to the testing machine. Al- $\mathrm{Zn}-\mathrm{Mg}$ alloys were investigated in this research which is subjected to a T6 heat treatment. The specimens are 
in the shape of cylindrical rods and contain as received microstructure and the bridges are in the shape of arc which are shown in literature [7]. To investigate multi-axial fretting fatigue behavior of Al-Zn-Mg alloys, specimens are exposed to different frequency from $3 \mathrm{~Hz}$ to $15 \mathrm{~Hz}$. These experiments are conducted based on cyclic stress controlled at equivalent stress amplitude of $252 \mathrm{MPa}$, a sine wave form and a stress ratio of $-1,200 \mathrm{MPa}$ Hertz contact stress imposed by fretting bridge, in lab air at temperatures of $22{ }^{\circ} \mathrm{C}$ and relative humidity of $40 \%$ [7].At the end of the experiment, the surface damage characterization and fretting fatigue fractures are observed by Quanta 200 FEG-SEM machine.

\section{Results and discussion}

\section{Effect of frequency on multi-axial fretting fatigue lives of Al-Zn-Mg alloys.}

The multi-axial fretting fatigue lives of Al-Zn-Mg alloy are shown in Fig. 1. As can be seen from Fig. 1, there is a certain relationship between the frequency and multi-axial fretting fatigue lives of Al-Zn-Mg alloys. Frequency and multi-axial fretting fatigue life is not a simple positive correlation or linear relationship. When frequency is $3 \mathrm{~Hz}$, multi-axial fretting fatigue lives of $\mathrm{Al}-\mathrm{Zn}-\mathrm{Mg}$ alloy is 18596 cycles which is the maximum. After that when frequency is increased to $9 \mathrm{~Hz}$, multi-axial fretting fatigue lives of $\mathrm{Al}-\mathrm{Zn}-\mathrm{Mg}$ alloys is 10051 cycles which is the minimum. Then when frequency is increased to $15 \mathrm{~Hz}$, multi-axial fretting fatigue lives of $\mathrm{Al}-\mathrm{Zn}-\mathrm{Mg}$ alloys is increased to 15477 cycles. That is, $9 \mathrm{~Hz}$ is the critical value of frequency, when the load frequency is less than the critical value fretting fatigue life decreases with increase of frequency, while the value is greater than the critical value fretting fatigue life will increase with increase of frequency. Effect of frequency on multi-axial fretting fatigue lives of $\mathrm{Al}-\mathrm{Zn}-\mathrm{Mg}$ alloys can be analyzed from the following three aspects: (1)When frequency is low, multi-axial fretting fatigue lives of Al-Zn-Mg alloy is longer because effect of chemical factors and other environmental factors on multi-axial fretting fatigue lives is large [8]. (2) The low frequency provides more extended time for crack propagation [9]. (3) Difference in frequency of the specimen will result in difference of friction coefficient on the specimen's surface which can lead to change of wear degree and fretting area of contact area of the specimen's surface, so as to change crack initiation and propagation, and further multi-axial fretting fatigue lives of $\mathrm{Al}-\mathrm{Zn}-\mathrm{Mg}$ alloy [10].

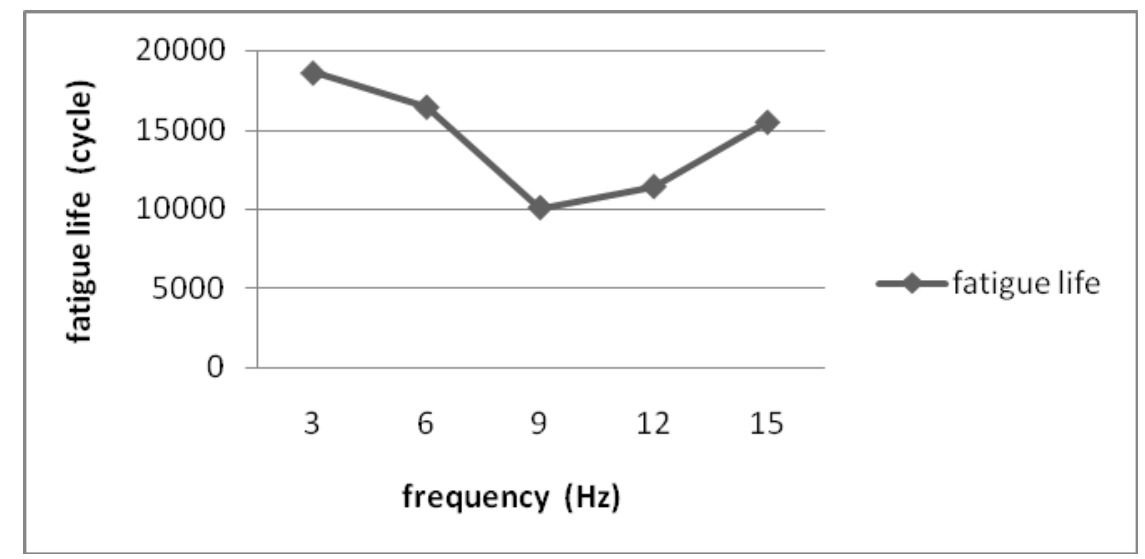

Fig.1 Fretting fatigue test results for Al-Zn-Mg alloy

The multi-axial fatigue and fretting fatigue lives of Al-Zn-Mg alloy at the same frequency are shown in Table 1. Identical in other conditions and frequency is $9 \mathrm{~Hz}$, the average multi-axial fatigue life of Al-Zn-Mg alloys is 370076 cycle while multi-axial fretting fatigue lives is 10051 cycle, compared with ordinary fatigue it was reduced by about $97.28 \%$. This shows that the fretting action will greatly speed up the fatigue damage and fatigue fracture of the specimen to shorten fatigue life of $\mathrm{Al}-\mathrm{Zn}-\mathrm{Mg}$ alloys. In the process of multi-axial fretting fatigue test of Al-Zn-Mg alloys, multi-axial fretting fatigue life is affected by multi axial stress, and influence of fretting wear on the surface of the specimen is also affected so that both of them reduce multi-axial fretting fatigue life. Fretting fatigue 
lives is lower than that of ordinary fatigue. Effect of fretting on multi-axial fretting fatigue life of Al-Zn-Mg alloys can be analyzed from the following two aspects: (1) Fretting action causes the contact surface area between the specimen and the fretting bridge foot to be subjected to repeated wear and tear so that new matrix is constantly damaged and oxidized and the serious stress concentration phenomenon has been produced in the contact area. The initiation and propagation of fatigue crack in the area are increased and the fatigue strength of the material is also reduced which leads to the greatly reduced multi-axial fretting fatigue life of Al-Zn-Mg alloys [11]. (2) In the process of fretting fatigue, fretting fatigue surface will produce adhesion, therefore, various damages can occur in fretting fatigue area of the specimen surface which can cause stress concentration on the boundary between sliding zone and sticking zone. Thus, fretting fatigue crack initiation in this region is more easily and it can lead to the early expansion of fretting fatigue crack, so that multi-axial fretting fatigue life is much lower than that of ordinary fatigue life [12].

Table 1 Comparison of lives between fatigue and fretting fatigue $(9 \mathrm{~Hz})$

\begin{tabular}{lccc}
\hline & Frequenc & Contact stress pressure & Fatigue lives /cycle \\
& $\mathrm{y}$ & & \\
\hline Fatigue & $9 \mathrm{~Hz}$ & $0 \mathrm{MPa}$ & 370076 \\
\hline Fretting Fatigue & $9 \mathrm{~Hz}$ & $200 \mathrm{MPa}$ & 10051 \\
\hline
\end{tabular}

\section{Analysis on multi-axial fretting fatigue damage characterization of Al-Zn-Mg alloys.}

The multi-axial fretting fatigue damage characterization of Al-Zn-Mg alloys is shown in Fig. 2. As can be seen from Fig. 2, the central region of fretting area and the boundary area are obvious where the central area is relatively flat and the edge area is rough. This is caused by the interaction between the specimen, the foot of the fretting bridge and the contact of the point contact. There are a lot of sliding, rolling, grinding and other traces at center area of fretting scars. This is due to at the early stage of fretting fatigue and under normal loading, the contact surface between the specimen and the bridge foot is in contact with the rough peak to cause adhesive wear [13]. Plastic deformation will be occurred in the contact surface between the micro convex bodies in the fretting area, under action of external force, the surface material is pressed to the sides which are perpendicular to the direction of movement to form convex bodies. Under the action of external force, convex body and asperity are caused rolling and continuous sliding and smooth. Under the action of oxidation, brittleness of plastic deformation layer on specimen surface can lead to part of the material to form wear debris. So, similar to the general fatigue, fretting fatigue damage will occur in the early adhesion wear.

Severe plastic deformation exists and there are obvious micro cracks in the fretting edge region in which these micro cracks are generally due to the contact stress. Combined effects of cyclic loading and surface friction, result of stress concentration is caused by phenomenon of stress concentration in the fretting zone of the specimen and the non- fretting area or the damage zone. The fracture position of the sample is located at the junction of fretting zone and non-fretting region because the junction is affected by contact stress, cyclic stress and surface shear stress. So there is serious stress concentration phenomenon which is beneficial to initiation and propagation of crack and lead to fracture of the specimen along edge of fretting damage zone. It can be clearly observed that each fretting damage zone is composed of three parts: slip region, partial slip zone, mixed zone. The edge position of fretting damage zone is the common existence of slip zone and mixed zone so that there are serious oxidation and wear in these area where there will generate black oxidized wear debris to render black. At the same time, there is a serious phenomenon of stress concentration in the area so that it is easy to crack initiation and propagation. 




Fig.2 SEM results of the fretting scars

(a) Morphology $(9 \mathrm{~Hz}),(b) \operatorname{Edge}(9 \mathrm{~Hz})$

\section{Analysis on multi-axial fretting fatigue fractures of Al-Zn-Mg alloys.}

Propagation area occupies proportion of the whole fracture is shown in Table 2. Whether it is general fatigue or fretting fatigue, higher frequency, the greater proportion of fatigue crack growth zone in the whole fracture zone and the smaller the final fracture zone. Correspondingly, lower frequency, there will exits smaller expansion area and the greater final fracture zone. This is related to the fatigue crack growth rate $d a / d N$, a large number of studies show that $[14,15]$, when $\Delta K$ (the amplitude of the stress intensity factor of the crack tip) is lower, basically unaffected by loading frequency. When $\Delta K$ is larger, frequency has a greater impact. When frequency is $3 \mathrm{~Hz}, d a / d N$ is large and fatigue crack growth rate is high. It can concluded that the greater distance from the crack propagation in the cyclic loading cycle, the smaller the crack growth stage, so that the proportion of final fracture zone in the area of the total area is large. When frequency is $15 \mathrm{~Hz}, d a / d N$ is small and fatigue crack growth rate is low. It can concluded that the smaller distance from the crack propagation in the cyclic loading cycle, the larger the crack growth stage, so that the proportion of final fracture zone in the area of the total area is small. When frequency is $9 \mathrm{~Hz}$, the crack growth zone size of general fatigue and fretting fatigue is very close, this shows that effect of fretting on fatigue crack growth zone is small. According to the analysis, due to fretting, fretting damage area formed on the surface of fretting fatigue specimen to cause serious stress concentration. Fretting fatigue crack is very easy to crack initiation and propagation. After crack initiation, the early extension is influenced by cyclic stress and surface friction (shear stress), expansion rate is very fast. But the extension is small, under usual conditions, the crack growth morphology is not observed. When extended to a certain depth, crack continues to expand and turn to the vertical direction of specimen's surface. At this time, shear stress almost does not work, the crack growth is mainly affected by the applied cyclic stress, at this stage (the later stage of crack propagation), fatigue and fretting fatigue crack growth is similarly affected by stress and frequency. Therefore, the propagation area accounted for the proportion of the whole fracture. 

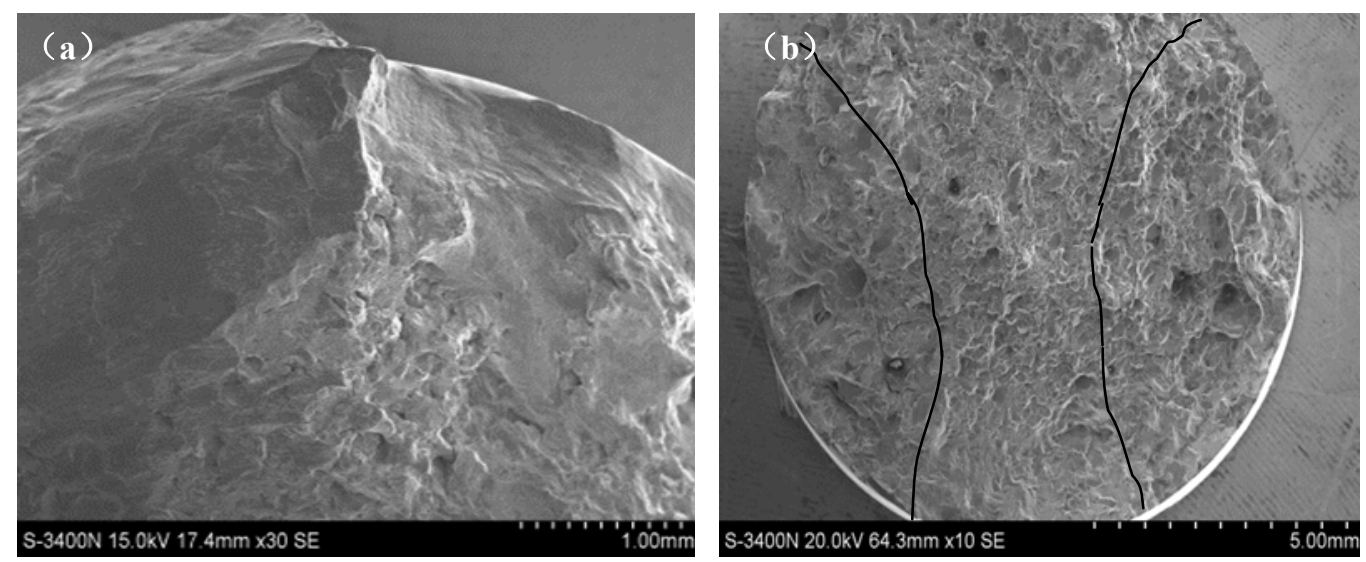

Fig.3 SEM results of fretting fatigue fractures $\left(\sigma_{\mathrm{a}}=252 \mathrm{Mp}\right)$

(a) Fatigue source region $(9 \mathrm{~Hz})$, (b) Full view, $(15 \mathrm{~Hz})$

Table 2 Propagation area occupies proportion of the whole fracture

\begin{tabular}{cccc}
\hline No. & $\begin{array}{c}\text { Frequenc } \\
\mathrm{y}\end{array}$ & Contact stress pressure & Expansion area size \\
\hline 1 & $3 \mathrm{~Hz}$ & 180 & $31 \%$ \\
\hline 2 & $9 \mathrm{~Hz}$ & 0 & $40 \%$ \\
\hline 3 & $9 \mathrm{~Hz}$ & 180 & $42 \%$ \\
\hline 4 & $15 \mathrm{~Hz}$ & 180 & $48 \%$ \\
\hline
\end{tabular}

The fatigue source region and the final fracture zone were tested by EDS analysis which is shown in Table 3. Analysis results show that: The microstructure near fatigue source region of the specimen is river pattern and the oxygen content is $53.61 \%$, while the microstructure in the vicinity of final fracture is a tough nest and the oxygen content is $4.37 \%$ which is far less than the oxygen content in the vicinity of the crack source area. This is due to multi-axial fretting fatigue test of Al-Zn-Mg alloys, the fretting damage area of the specimen is subjected to the combined action of contact stress, cyclic loading and surface friction. A serious stress concentration phenomenon can be caused in the area of fretting damage to produce delamination and repeated rolling, oxidation, in this way granular oxide abrasives $\left(\mathrm{Al}_{2} \mathrm{O}_{3}\right.$ wear debris $)$ can be formed to accelerated crack initiation and propagation, eventually lead to the final fracture of the sample. The source of fatigue crack often lies in the vicinity of fretting damage zone. In the process of fretting fatigue, partially oxidized wear particles are subjected to extrusion and sliding to enter the fracture zone, so that the fatigue source region of the fracture surface has a high content of oxygen content. Along with the crack extending from crack initiation region to propagation region and final fracture region, the number of oxidized particles in propagation and final fracture zone is reduced greatly. Therefore, the oxygen content in the surface area of the fracture surface is related to the distance from the specimen surface fretting damage zone along the crack propagation direction.

Table 3 Comparison of fractures' oxygen content at various location (wt\%)

\begin{tabular}{lcc}
\hline Element & Fatigue source region & Final fracture zone \\
\hline $\mathrm{C} \mathrm{K}$ & 0.78 & 1.24 \\
\hline $\mathrm{O} \mathrm{K}$ & 53.61 & 4.37 \\
\hline $\mathrm{Al} \mathrm{K}$ & 40.31 & 89.19 \\
\hline $\mathrm{Mg} \mathrm{K}$ & 0.69 & 1.48 \\
\hline $\mathrm{Zn} \mathrm{K}$ & 2.31 & 3.28 \\
\hline
\end{tabular}




\section{Summary}

1. Frequency and multi-axial fretting fatigue life is not a simple positive correlation or linear relationship. The fretting action will greatly speed up the fatigue damage and fatigue fracture of the specimen to shorten fatigue life of Al-Zn-Mg alloy.

2. Fretting fatigue damage will occur in the early adhesion wear because severe plastic deformation exists and there are obvious micro cracks in the fretting edge region in which these micro cracks are generally due to the contact stress.

3. Whether it is general fatigue or fretting fatigue, higher frequency, the greater proportion of fatigue crack growth zone in the whole fracture zone and the smaller the final fracture zone. The oxygen content in the surface area of the fracture surface is related to the distance from the specimen surface fretting damage zone along the crack propagation direction.

\section{Acknowledgments}

The authors are grateful to the National Natural Science Foundation of China (51201143) for financial support of this work.

\section{References}

[1] B. LIU, G. HE, X. JIANG, M. ZHU: Fatigue \& Fracture of Engineering Materials \& Structures Vol.34 (2011),p.974

[2] B. Liu, G.Q. He, X.S. Jiang,M.H. Zhu: Proceedings of the Institution of Mechanical Engineers, Part J, Journal of Engineering Tribology Vol.225(8)(2011), p.754

[3] Nowell D, Dini D, Hills D A: Engineering fracture mechanics Vol.73(2) (2006) , p. 207

[4] M Mirzajanzadeh : Engineering Fracture Mechanics Vol.78(2011) p.1233

[5] JA Alic, AL Hawley, JM Urey: Wear Vol.56.(1979)p.351

[6] Sachin R. Shinde, David W. Hoeppner: Wea Vol.61(3- 4) .(2006) p.426

[7] Jiang Xiaosong, He Guoqiu, Liu Bing, Liu Xiaoshan: Vol.41.(2012) p.136

[8] M. Mirzajanzadeh, T.N. Chakherlou, J. Vogwell: Engineering fracture mechanics Vol.78(46) .(2011) p.1233

[9] J.A. Alic, A.L. Hawley: Wear Vol.56.(1979), p.377

[10] J.M. Ambrico, M.R. Begley:International Journal of fatigue Vol.23(2) .(2001) p.121

[11] R.B. Waterhouse, B. Noble, G. Leadbeater:Journal of mechanical working technologyVol.8(2-3).(1983) p.147

[12] Sachin R. Shinde, David W. Hoeppner:Wear Vol.261(3-4) .(2006) p. 426

[13] Sanat Wagle, Hiroshi Kato: International Journal of Fatigue Vol.31(8-9) .(2009) p.1378

[14] Zhang you-hong, Lu guo-zhi,Li zhong: Acta Aeronautica et Astronautica Sinica Vol.28(2).(2007) p. 332

[15]Li Qiang,ZhouChangyu,Huang Wenlong:Journal of nanjing university of chemical technology Vol. 22(1).(2000) p. 32 